\title{
DETECTION OF LUNG-TISSUE
}

\author{
IN THE \\ EXPECTORATION OF PERSONS AFFEC'TED WITH \\ PHTHISIS.
}

BY

\begin{abstract}
SAMUEL FENWICK, M.D., ASSISTANT-PHYSICIAN TO THE CITY OF LONDON HOSPITAL FOR DISEASES OF THE CHEST; IATE IECTURER ON PATHOLOGICAL ANATOMY IN THE UNIVIRSITY OF DURHAM.
\end{abstract}

Received May 22nd.-Read June 26th, 1866.

Ir frequently happens that the physical examination of the chest fails to afford decisive evidence of tubercle in persons whose symptoms have led the physician to suspect its presence. Under such circumstances the examination of the expectoration by the microscope for pulmonary tissue has been recommended by various authors, as likely to be of the greatest service.

I have included in the following paper the results obtained from the microscopical examination of the sputa of 100 real or suspected cases of pulmonary consumption, in the hope of showing what advantages we may expect to derive from this method of investigation, and how far the microscope is capable of confirming or correcting the information furnished by auscultation and percussion. 
All the cases here reported were treated at the Victoria Park Hospital, either by myself or by my colleagues, and I take this opportunity of thanking them for their courtesy and kindness in granting me opportunities for observation which the out-patient department was unable to afford.

Dr. Andrew Clark has recommended that the expectoration should be poured upon a flat surface, and the particles suspected to consist of elastic fibre should be picked out with needles, and placed beneath the microscope.

Now, although large pieces of lung-tissue can be detected by this method, it is evident that the smaller fragments may be easily overlooked, and that consequently our inability to discover elastic fibre in this way is no sure evidence that none is present in any particular specimen of expectoration.

On this account I have adopted the plan of liquefying the sputa by means of pure caustic soda, when any particles of lung that may be contained in it fall to the bottom of the vessel, and can be removed and placed beneath the microscope.

In order to test the accuracy of this process I performed numerous experiments, of which the following is an example :

One tenth of a grain of lung-tissue was divided by needles into ten parts, and the $\frac{1}{100}$ th of a grain thus obtained was placed in the expectoration of a person affected with bronchitis, it having been proved by previous examination to contain no elastic fibre. The mucus was liquefied by boiling it with a solution of pure soda, and the mixture then poured into a conical=shaped vessel. The particle of lung was easily removed and recognised by the microscope.

But as the $\frac{1}{100}$ th part of a grain of lung consists of from twenty to thirty air-cells, and as the presence of elastic fibre can be determined with the greatest certainty by means of the microscope in half or even in a smaller part of a cell, it is evident that it might be possible in this way to detect the $\frac{1}{4000}$ th to $\frac{1}{6000}$ th part of a grain of pulmonary structure in the sputa of patients affected with phthisis. Such minuteness is, however, never requisite, as we need no more 
expect to meet with a part of an isolated air-cell in the expectoration than to be consulted by a patient with a single tubercle in his lungs. In one case $I$ counted in the mucus coughed up in only twelve hours, as many as 800 fragments of lung, and we generally find from fifty to sixty pieces even where, from auscultation, we should have expected but a small amount of destruction to be going on.

I have detected numerous fragments of elastic fibre in the mucus expectorated in only half an hour; but as the amount of lung expelled seems to vary at different times of the day, the only safe plan is to liquefy the whole of the sputa of twenty-four hours, or, if it is difficult to obtain the expectoration for so long a period, to examine what is coughed up during the night and early morning.

I have only once found, on a second examination, pulmonary tissue when I had on a previous trial failed to discover it. This was in the case of a patient affected with a cavity of long standing, and in such cases it will be wise for the physician to re-examine the expectoration after a few days' interval, if on the first examination he should fail to obtain evidence of the presence of the elastic fibre.

In phthisis there are, in reality, only two stages, viz., those of deposition of the tubercle, and of cavity ; for as soon as softening commences fragments of lung are expelled, and necessarily cavities are produced; but as the microscope should always be used as an adjunct to auscultation, I have grouped the cases contained in this paper under the three stages generally recognised by auscultators, viz., deposition, softening, and cavity.

\section{Stage of Deposition of Tubercle.}

The expectoration was examined by the microscope in twenty-four cases in which phthisis in its earliest stage was either suspected or was diagnosed by the stethoscope, and in thirteen of these fragments of lung-tissue were discovered. 
In seven of the twenty-four no physical signs of disease in the lungs were found, but the existence of tubercle was suspected either on account of the occurrence of hæmoptysis or from the history of the case showing an hereditary tendency to consumption in persons affected by cough, expectoration, and loss of flesh; no elastic tissue could be found in the sputa of any of them.

The microscope therefore failed as completely as auscultation in proving the existence of phthisis in this difficult class of cases, and I cannot help suspecting that in many of these the symptoms arise rather from the general derangement of health preceding the formation of tubercle than from an actual deposit in the pulmonary tissue.

Fig. 1.

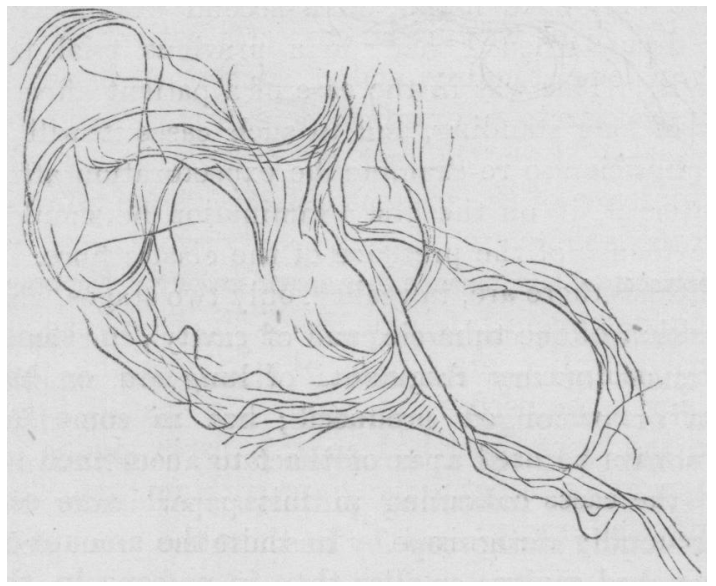

Portion of lung-tissue as it appears under the microscope.

In sixteen cases auscultation and percussion afforded indications sufficient to justify the suspicion of the existence of tubercle, and in thirteen of these lung-tissue was found in the expectoration. Of the cases in which it was absent one had never suffered from hæmoptysis, and the physical indioations were a doubtful deficiency of resonance under the right clavicle, with feeble inspiration, rhonchus existing in other parts of the chest. Another had been ill six years, 
and there was deficient resonance under the right clavicle, with feeble inspiration. The third had suffered from hæmoptysis, and presented harsh inspiration and prolonged expiration under the right clavicle.

In the cases in which lung-tissue was found in the sputa some presented deficient resonance on percussion under the

Fig. 2.

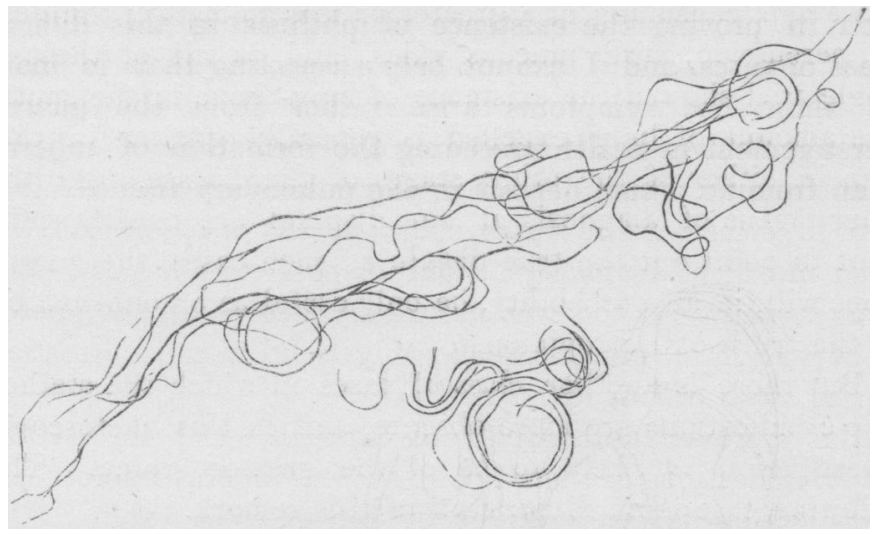

Fragments of lung-tissue as they appear under the microscope.

clavicle or in the supra-scapular region. In some there were "clicks of dry rhonchus," diminished or harsh inspiration or prolonged expiration, and in some increased vocal resonance in the apex of the lung, combined with the general symptoms indicating phthisis. All were examined with the double stethoscope. In these the amount of lungtissue coughed up was smaller than in persons in the later stages of the disease, and the proportion of fragments of isolated air-cells was unusually large, and the pieces of bronchial tubes were of small size.

The microscope is therefore in this stage a most valuable adjunct to auscultation, inasmuch as it shows that in the majority of cases in which physical signs of the existence of tubercle are present softening of the deposit has already commenced, and lung-tissue in small quantities is being expelled. Nor is it of less value when it proves that no 
ulceration is taking place, for such a fact should induce us to look hopefully upon such a case, and should encourage us to persevere in our endeavours to ward off attacks of inflammation.

\section{Stage of Softening.}

In twenty-four cases there were well-marked signs of this stage, consisting of a greater or less amount of dulness on percussion, with mucous râles, and in all of these a single trial showed the existence of lung-tissue in the sputa. The amount of lung expelled is generally greater in this than in either of the other stages of the disease. As the other means of diagnosis at our disposal are usually sufficient to point out the true nature of such cases, the microscope will, in all probability, be only useful as a confirmation of the truth of our opinion.

But there is another class of cases in which the stethoscopic indications are more obscure, and in this microscopic investigation is likely to be of the greatest value. The following case is an illustration of this remark.

CASE 1.-A man æet. 27 applied at the hospital, complaining of cough, copious expectoration, severe sweatings, and rapid loss of flesh. He had only felt ill for two months, but his pulse was very quick and his voice whispering.

There was no dulness on percussion, but a dry rhonchus was heard on the whole of the anterior part of the chest, and mucous râles were present at the base of each lung. The microscope at once showed the nature of the case by detecting sixty fragments of pulmonary tissue in the mucus expectorated during only five hours.

There is, again, another class which often causes much doubt and anxiety to the practitioner, viz., where rapid loss of flesh and severe night-sweats take place in a person who has for many years been affected with chronic bronchitis. Here the existence of emphysema often masks any dulness on percussion that might otherwise be produced by the tuber- 
cular deposit, and auscultation fails to afford decisive evidence as to the actual nature of the disease. Of this the following case is an example.

Case 2.-A man about 40 years of age applied to the hospital with cough, expectoration, and rather rapid loss of flesh and strength.

He had been for four years subject to bronchitis in the winter, but had each summer been relieved, and he felt confident that as soon as the warm weather returned his symptoms would be again alleviated. He had never had hæmoptysis. There was no distinct dulness, and the cardiac region was very resonant on percussion. There was loud and harsh inspiration, both at the front and the back of the chest, with mucous râles below the clavicle. The microscope showed forty-three pieces of lung-tissue in the expectoration.

I examined the sputa of fifteen cases in which auscultation afforded doubtful evidence of the existence of tubercle, and in seven I found pulmonary tissue. On the average, the proportion of bronchial tubes amounted to 14 per cent. of the fragments of lung-tissue in the expectoration. It is worthy of remark that this is nearly the same proportion as was found in the cases which are considered by stethoscopists to be in the stage of "deposition of tubercle," whilst where decided dulness and mucous râles existed the proportion of tubes formed only 9 per cent. May not this difference arise from the tubercle being more deposited in the former class in the mucous membrane of the tubes themselves, whilst in the latter the air-cells are more exclusively affected?

But if the presence of pulmonary tissue in the expectoration of patients in this stage be so general, it will be readily seen that its absence is a most important fact in the history of any particular case. To show the practical bearing of this, I have added the following case.

Case 3.-A woman five montlis pregnant was admitted 
an out-patient of the hospital, complaining of severe cough, expectoration, and great loss of flesh and strength. I thought $I$ detected a little deficiency in the resonance of the right sub-clavicular region, and there were some mucous râles on the left anterior chest, with harsh inspiration at the base of each lung. I could find no lung-tissue in the expectoration, but her general symptoms were so threatening that $I$ re-examined it a fortnight afterwards, and with the same result.

She steadily improved under treatment, and at her last visit reported that her cough and expectoration had both ceased.

\section{Stage of Cavity.}

In thirty-five cases the stethoscope detected signs of cavities in the lungs, and in every instance there was lungtissue in the expectoration. In one case I failed to find any on the first trial, but on repeating the experiment a week afterwards, with another specimen of the sputa, ten fragments were discovered.

I was disappointed in thus finding elastic fibre in every case, as in a considerable number of the patients the general health had so greatly improved, and the expectoration had so altered in character and decreased in quantity, that I had hoped to have been able to prove the complete arrest of the ulceration to be not infrequent. Two persons with stethoscopic indications of cavities in the lung, whose cases are not included in this paper, denied that they had any expectoration at all.

One patient stated that he only expectorated a little mucus for about half an hour each morning, but even this, when examined, was found to contain twenty fragments of lung. Another coughed up only a little thin semi-transparent mucus in the mornings, and in it were contained eleven pieces of cell-structure and a bronchial tube.

But if the expulsion of the elastic fibre of the lungs is so general where cavities exist, it will be easily seen that 
the microscope is likely to prove of value in the diagnosis of enlarged bronchial tubes. I carefully examined the expectoration of two persons in whom I believed this condition to be present, and in neither case could $I$ discover any fragments of lung.

As in all the stages of consumption we find portions of lung in the sputa, it will be interesting to ascertain whether there is any difference in the size of the pieces expelled in different periods of the disease.

In order to discover this I carefully counted the size of the fragments in sixty-nine cases, and from these the following table has been formed.

Table showing the size of the fragments of lung contained in the expectoration of sixty-nine cases of consumption.

\begin{tabular}{|c|c|c|c|c|c|}
\hline Stage according to auscultation. & $\begin{array}{c}\text { Fragments } \\
\text { of } \\
\text { bronchial } \\
\text { tubes. }\end{array}$ & $\begin{array}{l}\text { Fragments } \\
\text { of } \\
\text { single air- } \\
\text { cells. }\end{array}$ & $\begin{array}{l}\text { Fragments } \\
\text { of from } \\
\text { one to six } \\
\text { air-cells. }\end{array}$ & $\begin{array}{c}\text { Fragments } \\
\text { above the } \\
\text { size of six } \\
\text { air-cells. }\end{array}$ & $\begin{array}{c}\text { Large } \\
\text { fragments. }\end{array}$ \\
\hline $\begin{array}{l}8 \text { Cases of deposition of } \\
\text { tubercle with well- } \\
\text { marked physical signs }\end{array}$ & 15 p.c. & 41 p.c. & 34 p.c. & 10 p.c. & - \\
\hline $\begin{array}{l}7 \text { Cases of softening stage } \\
\text { without marked dul- } \\
\text { ness on percussion }\end{array}$ & 14 p.c. & 33 p.c. & 43 p.c. & 7 p.c. & 3 p.c. \\
\hline $\begin{array}{l}22 \text { Cases of softening with } \\
\text { decided dulness on } \\
\text { percussion. }\end{array}$ & 8 p.c. & 32 p.c. & 46 p.c. & 12 p.c. & 0.5 p.c. \\
\hline 32 Cases of cavity & 18 p.c. & 31 p.c. & 37 p.c. & 12 p.c. & 2 p.c. \\
\hline
\end{tabular}

It will be remarked that the proportion of bronchial tubes expectorated is smallest in the cases of the second stage, in which well-marked dulness showed the cellular structure of the lung to have been greatly implicated. It is greatest in those in which the signs of cavities existed, probably from the tubes which had formerly escaped destruction being in this stage attacked by the disease. Where 
cavities are present the large size of the tubes present in the sputa at once attracts the attention of the observer.

It might be supposed that there would be a great proportion of fragments of cells and an absence of larger pieces of lung in the stage of deposition, where the cavities formed are too small to give rise to the sound of mucous râles, and this the above table shows to be the case.

The proportion of fragments varying from one to six cells is greatest in the softening stage, and we can well imagine that cavernules likely to produce the crepitations so generally heard at this period would be the result of the expulsion of fragments of this size. I have usually found that the size of the fragments of lung coughed up in each case varies according to the sounds heard by the stethoscope; the finer the crepitation, the larger the proportion of the smaller pieces.

The appearance of the elastic fibre itself also varies in different cases. In some it is remarkably thin, whilst in others it is very coarse, granular, and opaque. This latter appearance is generally found in chronic cases, and especially in those with well-marked signs of cavities. I have met with it in post-mortem examinations in the walls cf old cavities, and I believe it to indicate previous inflammation of the fibrous structure of the air-cells.

In order that the examination of the expectoration for lung-tissue should be successful considerable care is requisite. A solution of pure caustic soda should be kept ready, about fifteen grains to the ounce of distilled water, and equal parts of this and of the sputa should be used. This mixture should be boiled for two or three minutes in a wide beaker, over a rose gas-burner or spirit lamp, being occasionally stirred with a glass rod. When dissolved it is to be poured into a large conical-shaped glass, and three or four times its bulk of cold distilled water is to be gradually added. It may be then set aside, in order that all the particles not capable of solution in the soda may subside.

The deposit should be placed in very shallow cells for examination. The cells should be cut out of vulcanite of 
the thickness of an ordinary visiting-card, and should be fixed to glass slides with cements capable of resisting the action of alkaline solutions. If deeper cells are used there is a great probability that many of the fragments of lungtissue may be overlooked.

I generally use a microscope fitted with a double arm, carrying an inch-and-a-half and a half-inch object-glass, with the tube drawn out to its fullest extent, and having a No. 2 eye-piece. The intention of this arrangement is, of course, to obtain as large a field as possible, conjoined with sufficient magnifying power.

The chief precaution to be observed is perfect cleanliness of all the apparatus, for the elastic fibre clings firmly to glass. The dipping-tube should be therefore always both brushed out and washed after each examination, or a fresh one should be used on the next occasion.

There is no difficulty in distinguishing the elastic tissue of the lung from other matters that may exist in the expectoration. It is often found clinging to filaments of hair, silk, \&c., but in all cases the fineness of the fibres, their regularity and dichotomous division, render detection certain and easy.

The accompanying drawings of the elastic tissue of the lungs, after being boiled in solution of soda, will, perhaps, be useful to those not accustomed to the examination of expectoration in cases of phthisis. 